\title{
Spectroscopic studies of individual plasmon resonant nanoparticles
}

Jack J. Mock, David R. Smith, Mladen Barbic, Steven J. Oldenburg, David A. Schultz, et al.

Jack J. Mock, David R. Smith, Mladen Barbic, Steven J. Oldenburg, David A. Schultz, Sheldon Schultz, "Spectroscopic studies of individual plasmon resonant nanoparticles," Proc. SPIE 5221, Plasmonics: Metallic Nanostructures and Their Optical Properties, (28 October 2003); doi: $10.1117 / 12.506523$

SPIE Event: Optical Science and Technology, SPIE's 48th Annual Meeting, 2003, San Diego, California, United States 


\title{
Spectroscopic studies of individual plasmon resonant nanoparticles
}

\author{
J.J. Mock ${ }^{\mathrm{a}}$, D.R. Smith ${ }^{\mathrm{a}}$, M. Barbic ${ }^{\mathrm{b}}$, S.J. Oldenburg ${ }^{\mathrm{c}}$, D.A. Schultz ${ }^{\mathrm{a}}$, S. Schultz \\ ${ }^{a}$ University of California San Diego, 9500 Gilman Drive, La Jolla, CA, USA 92093-0319 \\ ${ }^{\mathrm{b}}$ California Institute of Technology, Pasadena, CA, USA \\ ${ }^{\mathrm{c}}$ Seashell Technology LLC, La Jolla, CA, USA
}

\begin{abstract}
We present a detailed description of the apparatus and techniques that we have utilized in our experimental study of individual plasmon resonant nanoparticles, along with a brief description of some major results. The apparatus consists of a spectroscopic system combined with a modified darkfield microscope, which enables the user to sequentially select individual resonant nanostructures in the microscopic field of view for spectroscopic study. Plasmon resonant nanostructures scatter light elastically, and typically have very large scattering cross-sections at their resonant optical wavelengths. In general, spectra can be obtained with acquisition times between .1 to 30 seconds, and color images can be captured using consumer digital color cameras. Spheres, tetrahedrons, and pentagonal platelets were fabricated using colloidal chemistry techniques. To produce highly anisotropic structures such as nanorods and "barbells", templates were used. Many of these nanostructures have been individually spectroscopically characterized, and their spectra correlated with their shape and size as determined by transmission electron microscope (TEM). The unique shape, size, composition, and dielectric surroundings of the individual plasmon resonant nanostructures determine their plasmon resonant behavior. We will show how the composition of the substrate on which the particles are immobilized and the dielectric of the surrounding medium have a significant effect on the plasmon resonance of the individual particles.
\end{abstract}

Keywords: Plasmon resonance, nanoparticles, nanorods, silver enhancement, colloid, barbells

\section{INTRODUCTION}

There has been great recent interest in the optical properties of gold and silver nanoparticles with at least one geometrical dimension below $100 \mathrm{~nm}$. At optical wavelengths, the free electrons in the metal particles can be collectively excited into a plasmon resonance that is strongly coupled to the incident electromagnetic radiation. Under dark field or evanescent (T.I.R.) white light illumination, the individual Plasmon Resonant Particles (PRPs) appear as bright, colored, diffraction limited "point source" scatterers. The plasmon resonance spectra of the nanoparticles is very sensitive to their shape [1-9], size [1,10,11], composition [12,13], local dielectric surrounding [14-16] and interparticle interactions [1720]. These PRPs have been used for optical coding [21], biological labeling [11, 22], biosensing [23-26], and for novel plasmonic devices and materials [27-31]. While many experimental studies have examined the properties of collections of PRPs (whether in solutions or in arrays), we have focused our efforts on the spectroscopic characterization of individual PRPs. We characterize the optical properties of the particles with a specially modified Nikon Labophot darkfield microscope that can capture the spectrum from individual PRPs. These spectra can be correlated with transmission electron microscope (TEM) images, thereby illuminating the relationship between optical properties and nanostructure. We have studied the plasmon resonance spectrum from hundreds of gold and silver particles of a variety of shapes, sizes, compositions and in a variety of local environments. These results provide insight into the optical properties of the PRPs which will contribute to the advancement of applications utilizing them individually and in more complex nanostructures.

\subsection{Apparatus}

\section{METHODOLOGY}

To image PRPs, we utilize a Nikon Labophot Darkfield microscope with 5x, 20x, 50x, and 100x Brightfield/Darkfield (BF/DF) objectives and a 100x oil immersion objective (Figure 1: and also described in reference [1]). Our source of illumination is a $75 \mathrm{~W}$ Xenon bulb, housed in a columnating/focusing assembly from Oriel. The Xenon light source is preferable as it has a smooth emission across the visible region of the spectrum, allowing for the excitation of plasmon resonances from $380 \mathrm{~nm}$ to $700 \mathrm{~nm}$. Sample illumination can occur via epi illumination (brightfield), or as is most common utilizing the appropriate darkfield cube through the darkfield microscope objective. Brightfield illumination is edited by Naomi J. Halas, (SPIE, Bellingham, WA, 2003) · 0277-786X/03/ $\$ 15.00$ 
only useful for viewing PRPs when using an oil immersion objective and a transparent substrate mounted to a "light sink" (such as a glass prism) to prevent backscattering. An alternate illumination is through a coupled $1 \mathrm{~mm}$ diameter multimode optical fiber with a compound output focusing lens. The fiber can be used to illuminate the sample from below in total internal reflection (TIR) or from above (at a large enough angle to result in darkfield). The lamp output is coupled into the optical fiber using a standard fiber-coupler (Oriel). Passband illumination can be achieved by positioning 10nm bandpass filters (Omega Optical) along the illumination light path. Samples of interest are mounted on a quartz right angle prism, to allow for TIR illumination on substrates such as glass slides and coverslips (placed with index matching oil). The samples are positioned with a high precision, (1 $\mu \mathrm{m}$ resolution) 3 -axis microscope stage (Prior). In order to study the polarization dependence of some samples, a rotation stage is mounted between the microscope stage and the prism allowing for the sample to be rotated with respect to a uni-directional polarized fiber illumination. PRPs are bright enough to be readily observed individually through a 50x or a $100 \mathrm{x}$ magnification objective. Selection of individual particles for spectroscopic characterization is done using a Nikon adjustable image plane aperture and monocular. While observing the image through the monocular, the particle is positioned within the selected aperture using the precision Prior stage. Light from this selected nanoparticle can then be directed to the spectrometer (Spex $270 \mathrm{M}$ ), using a fixed mirror and re-focusing lenses, all of which are mounted above the microscope. The spectrometer is equipped with two precision controlled gratings $(1200 \mathrm{~g} / \mathrm{mm}$ and $150 \mathrm{~g} / \mathrm{mm})$, and a Princeton Instruments CCD detector (1300 x 1030). Spectra are always normalized with a white scatterer (Labsphere) and background scattering (of substrate without PRP or spurious scatterers) is subtracted for all data presented.

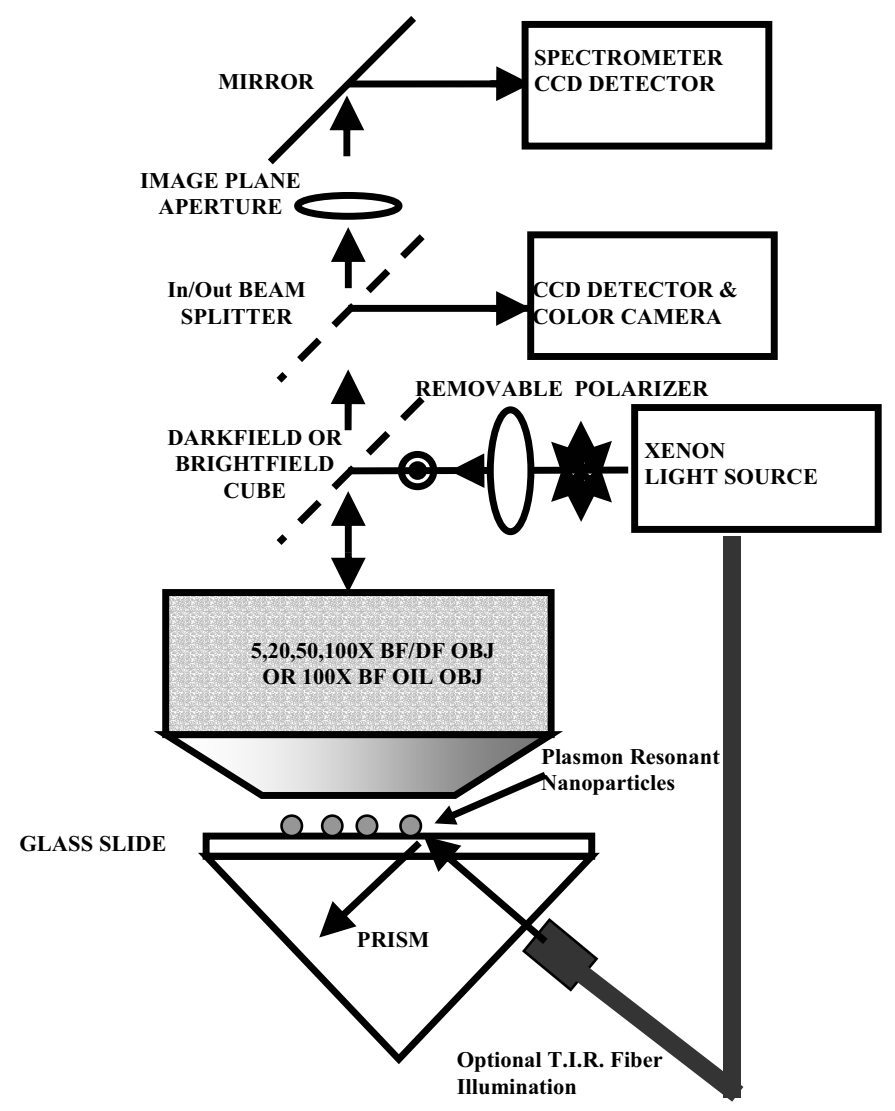

Figure 1: Darkfield Microscope and Spectrometer Apparatus

An additional CCD detector (Princeton Instruments CCD camera or a Nikon Coolpix 995) is also used on a secondary microscope output port for optional imaging of the nanoparticles. All Transmission Electron Microscope (TEM) work was done on a JEOL 2000 at the National Center for Microscopy and Imaging Research. This TEM was equipped with a sample tilt mechanism which made possible the determination of 3-D nanoparticle structure [1]. 


\subsection{Sample Preparation}

There are a variety of different techniques that can be used to produce plasmon resonant structures with dimensions less than $100 \mathrm{~nm}$ such as e-beam lithography $[5,17,21]$, nanosphere optical lithography [15, 24], and wet chemistry $[2,3,4$, $9,12,32]$. For single particle optical characterization studies, wet chemistry methods are advantageous since, under correct conditions, a variety of shapes can be produced in a single fabrication reaction. Typically, we produce $\mathrm{Ag}$ colloids by silver enhancing (BBI Silver Enhancer Kit) commercially available Au colloids (BBI) [1, 22]. The silver enhancement is a robust technique that can be used to silver coat nucleating Au colloids with diameters ranging from a few nanometers to over $100 \mathrm{~nm}$. We have successfully produced PRPs from gold particles that are suspended in solution, immobilized on a substrate, or embedded in a biological tissue [22]. In solution, we typically suspend a desired number

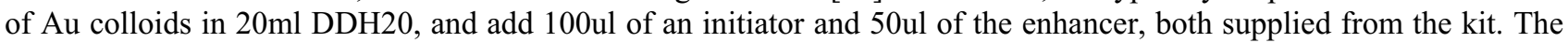
amount of enhancer and the number of Au colloids determine the final size of the resulting Ag colloid. We determine the approximate size of the Ag colloids by a measuring the optical extinction of the solution, in a Cary Spectrophotometer. The Ag colloid solution is typically composed of a variety of particle shapes that can be individually studied, including spheres, pentagons, tetrahedrons and aggregates (Figure 2A).

Nanorods of $25 \mathrm{~nm}$ to $50 \mathrm{~nm}$ diameter are produced with a templated plating technique [6,13]. A gold film is sputtered ( 15 minute at $15 \mathrm{mTorr}$ and $15 \mathrm{~mA}$, Technics Hummer V) on one side of a porous $7 \mu \mathrm{m}$ thick polycarbonate membrane (Costar, Nuclepore $15 \mathrm{~nm}$ pore size). The membrane is then placed Au side down within a custom jig which contacts the $\mathrm{Au}$ layer with a conductive surface and is filled with an electroplating solution to cover the top surface of the porous membrane. An electric potential is established between the bottom Au layer and the top solution, and the resulting current determines the amount of plated metal that will fill the pores in a given amount of time. We use this technique with gold or Nickel plating solutions (RTU solutions, 434 gold and high speed nickel sulfamate, Technic Inc.) to create
A) $\mathrm{Au}$ colloid
Silver enhanced colloid
Ag colloid under darkfield microscope

B)
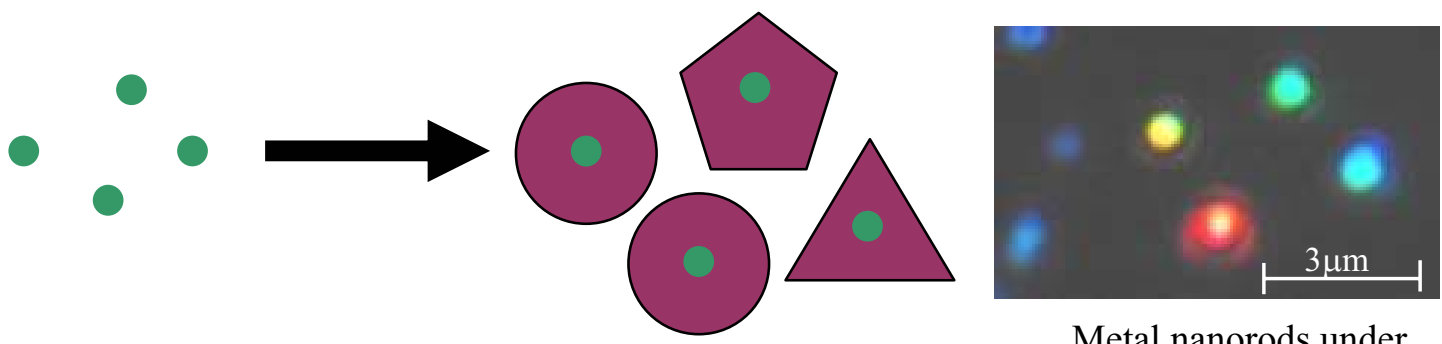

Metal nanorods under

Templated plating

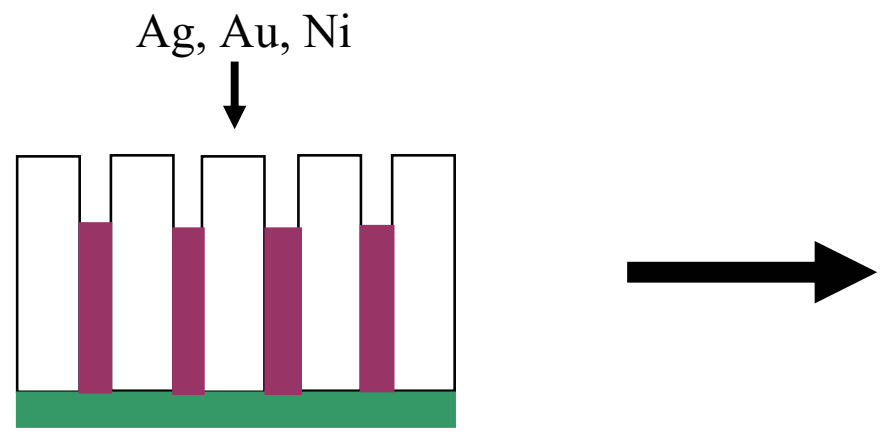

darkfield microscope

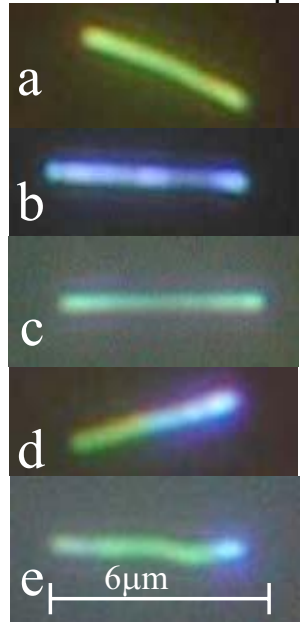

Figure 2: Fabrication of plasmon resonant nanostructures and color CCD images of far field scattering of completed PRPs under Darkfield microscope. A) Silver enhancement of Au nucleating colloids. B) Templated plating to produce plasmon resonant nanorods (a. gold, b. silver, c. nickel, d. gold/silver, e. nickel/gold/silver) 
gold or nickel nanorods. Silver nanorods are grown using an electroless silver plating technique where the silver enhancement kit is used in place of the electroplating solution, and no current is required. However, using the electroless silver enhancement requires that the silver segments always follow a gold layer, as the silver enhancer does not nucleate on nickel. By exposing a membrane sequentially to different plating solutions, it is relatively easy to grow nanorods with multiple segments consisting of different metals with each metal segment having a characteristic plasmon resonance and a desired length. Once the desired nanorod length and composition is obtained, the membrane is removed from the jig and is dissolved in chloroform. The nanorods themselves are then suspended in solution and can be immobilized on a substrate for optical and TEM analysis (Figure 2B). The preferential illumination of the nanorods is to use a 100x oil immersion bright field objective, with the nanorods (embedded in 1.5 index oil) immobilized on a glass slide, mounted on a right angle prism (to act as a "light sink", see references 6 and 13). The plasmon resonance of the nanorods is polarization dependent. The nanorod cross section $(25 \mathrm{~nm}$ to $50 \mathrm{~nm})$, is the plasmon resonant dimension in all of our nanorod studies. Therefore, the illumination must be polarized perpendicular to the rod length in order to preferentially excite this mode, allowing for the unique size and composition dependent optical scattering signature for each segment to be observed. Polarization of the illumination along the rod length (typically $>1 \mathrm{~m}$ ) results in the excitation of a broad white scattering mode.

We have also experimented with new sample preparation techniques, such as the silver enhancement of composite nanorods. For example, composite nanorods with a gold/nickel/gold pattern, produced using the electroplating technique described above, were silver enhanced. The gold segments preferentially nucleate the silver enhancer solution resulting in two Ag nanoparticles linked by a nickel nanorod (Figure 3). In the case shown below, the electroplated nanorods are immobilized on a substrate prior to enhancement, after which a drop of the enhancer solution is placed over the substrate for a controlled length of time before rinsing with DDH20. This enhancement technique could potentially be extended to create more complicated structures such as necklaces of $\mathrm{Ag}$ particles. Precisely spaced pairs or chains of plasmon resonant nanoparticles may be useful as SERRS substrates and for plasmon light propagation.
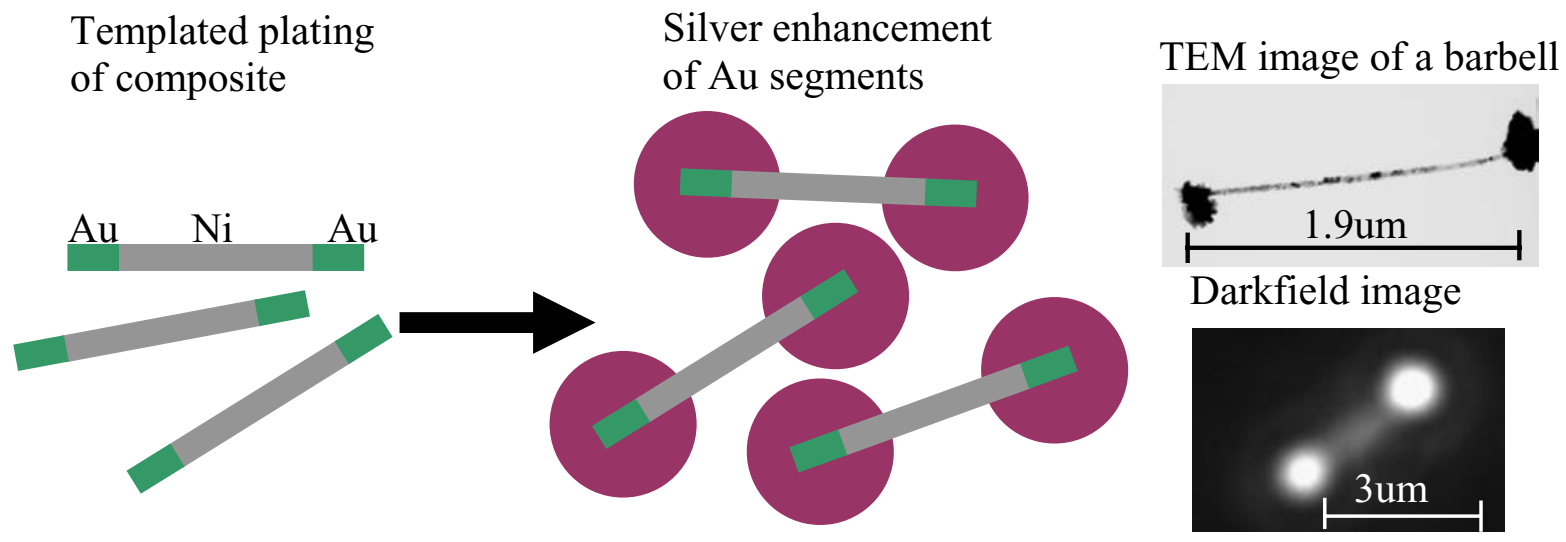

Figure 3: Representation of fabrication technique used to create controllably spaced plasmon resonant nanostructures, including TEM and darkfield microscope images of typical "barbell" nanostructures.

All nanoparticles are immobilized on substrates prior to optical characterization. This is typically accomplished by placing a drop of the colloidal solution on the substrate and letting it evaporate, or by first treating the substrate with positively charged poly-lysine and binding the colloids prior to rinsing the surface with DDH20. Substrates we routinely use that are compatible with our illumination scheme (i.e. low background) include glass and quartz slides and coverslips, silicon wafers with and without oxide layer, silicon nitride TEM windows (Structure Probe Inc.), formvar and carbon coated copper TEM grids (Ted Pella) and plastic multi-well ELISA plates. 


\section{DATA}

\subsection{Shape Dependence}

Correlating optically characterized PRPs with TEM for geometrical analysis, which we call "mapping", provides insight into the effects of shape and size on the plasmon resonance of the nanoparticles. We have mapped hundreds of PRPs immobilized on formvar coated TEM grids or SiN TEM windows (for temperature annealing studies). The results of our mapping experiments on colloidally prepared Ag [1] show that spherical particles preferentially scatter blue light $(400 \mathrm{~nm}-500 \mathrm{~nm})$, pentagons scatter green light $(500 \mathrm{~nm}-550 \mathrm{~nm})$, and tetrahedrons scatter red light $(550 \mathrm{~nm}-750 \mathrm{~nm})$. In addition to the shape effect, the spectrum of a particle tends to red-shift (and broaden in the case of spheres) with increasing size. Figure 4 shows the results of a typical mapping experiment for 3 individual PRPs. The spectra obtained from individual Ag PRPs correlate well with other published shape dependence results $[2,3,4,7,8]$. In addition to Ag colloids, we have optically characterized individual Au colloids (BBI) ranging in diameter from $40 \mathrm{~nm}$ to $100 \mathrm{~nm}$ and ranging in size dependent plasmon resonance peak position from $520 \mathrm{~nm}$ to $570 \mathrm{~nm}$ respectively. These colloid solutions are of high uniformity (coefficient of variation $<20 \%$ ), and consist primarily of spherically shaped nanostructures.

The mapping technique described here allows for iterative characterization of individual nanoparticle samples between the optical microscope and further modification or characterization. Using this technique, we have been able to alter the environment of the PRPs and follow the resulting changes in the spectra and morphology of the individual PRPs. As an illustration of this technique, we present the results of one such heating study in Figure 5. By heating the $\mathrm{Ag}$ nanoparticle bound to a silicon nitride substrate, using a standard hot plate as described in reference [1], the shape of the tetrahedral PRP could be subtly altered, as determined by TEM characterization, while monitoring the spectral properties on the optical microscope. The results show that as the corners of the tetrahedron are rounded, the plasmon resonance is incrementally blue-shifted. Tuning the properties of an individual PRP in this manner is insightful, and confirms a sensitivity to shape that would be difficult to discern in ensemble studies. For example, this study shows that the plasmon resonance of a tetrahedron is strongly influenced by the integrity of the vertices, as has also been described in other publications $[2,7,8]$.

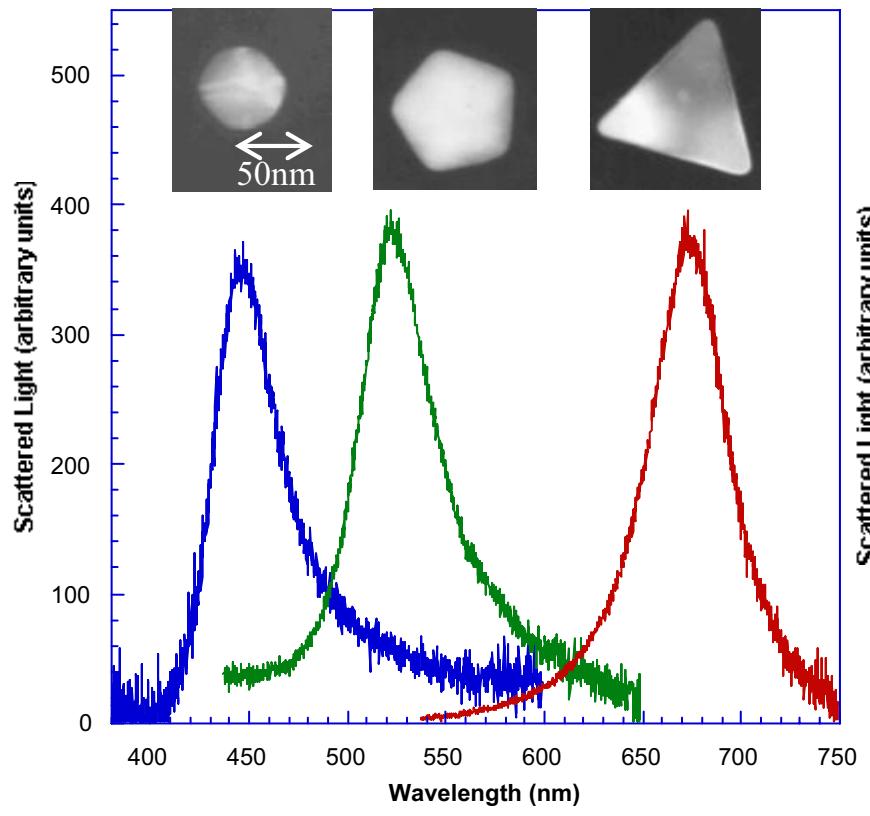

Figure 4: Typical spectral results of Ag nanoparticle mapping. Generally, spheres scatter in the blue, pentagons scatter green, and tetrahedrons scatter red light.

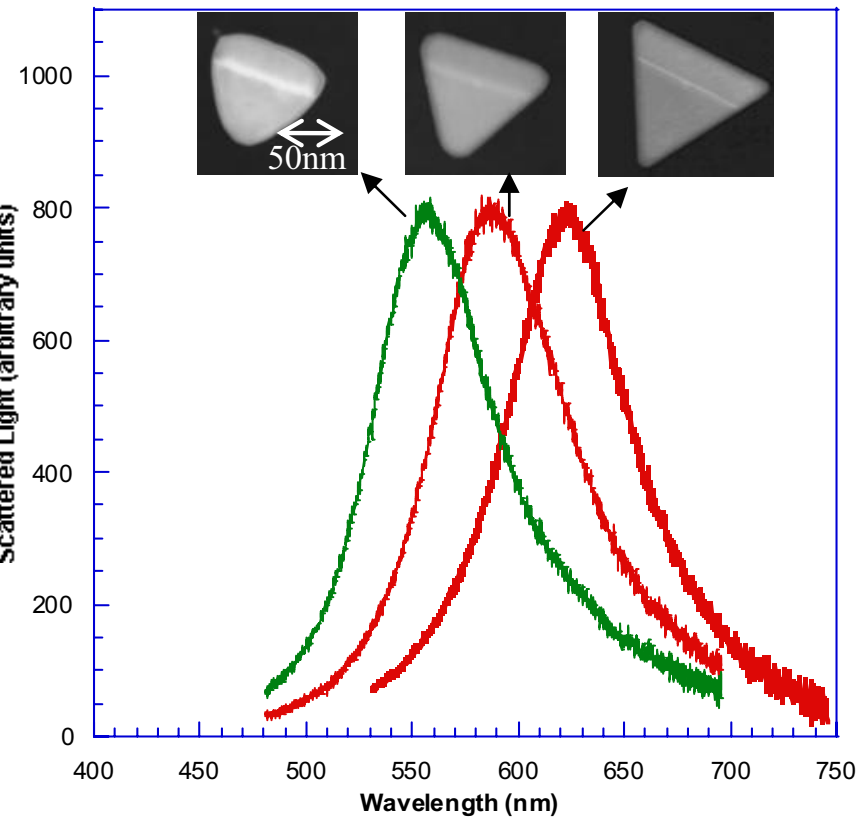

Figure 5: Tuning plasmon resonance of individual Ag nanoparticle. The tetrahedron is repeatedly heated and the changing particle's morphology and spectra are mapped to show their correlation. 
Other shapes, such as nanorods [6,13], (as shown in Figure 2), aggregates of 2 or more colloids [17, 18, 19], and other asymmetric forms such as ellipses [5, 17] display polarization dependent optimal signatures, and provide a further avenue for plasmonic nanostructure design.

\subsection{Filtered Optical Imaging of Nanostructures}

The optical microscope we use to characterize individual PRPs is equipped with a 100x magnification chromatically corrected objective, which allows for diffraction-limited imaging of the scattered light from the plasmon resonant nanoparticles. Images are recorded by a CCD camera (Princeton Instruments). Each nanoparticle in a particular far field CCD image area appears as a bright diffraction-limited spot with a characteristic point spread function (Figure 2A). For the 100x magnification, the typical point spread function has an F.W.H.M. of $\sim 8$ pixels. A cross-section of the point spread function for a given PRP has a maximum at the center position of the scatterer and can be used to locate the position of the particle with respect to its local environment, with nanometer scale resolution [22]. Of course, it is not possible to determine the distance between two closely spaced identical PRPs with nanometer precision, as the point spread functions of the two PRPs will generally interfere. However, if the two PRPs are distinct spectrally, then even this limitation can be overcome. This point is illustrated in Figure 6. Two closely spaced PRPs appear as a single bright scatterer (Figure 6 insert) under white light illumination. The pair in Figure 6 is composed of a spherical particle (blue scatterer) and a tetrahedron (red scatterer) spaced $225 \mathrm{~nm}$ apart-a scale below the resolution limit of an optical microscope, but large enough that inter-particle interactions are not dominant. In order to determine the relative positions of the two PRPs without the use of an electron microscope, we take advantage of the fact that the resonances of the two PRPs are spectrally well separated. By inserting $10 \mathrm{~nm}$ bandpass filters (Omega) into the illumination path, we are able to measure the point spread function of one particle while rejecting the point spread function of the other, thus increasing the interparticle contrast. To measure the interparticle distance, we take line-scans through two consecutive CCD images of the same particle(s), the first taken with a $410 \mathrm{~nm}$ centered $10 \mathrm{~nm}$ bandpass filter (at the resonance of the spherical nanoparticle) and the second with a $650 \mathrm{~nm}$ centered bandpass filter (at the resonance of the tetrahedron). By comparing the pixel peak positions of each line-scan, we arrive at a measurement of the relative position between the two PRPs. It is
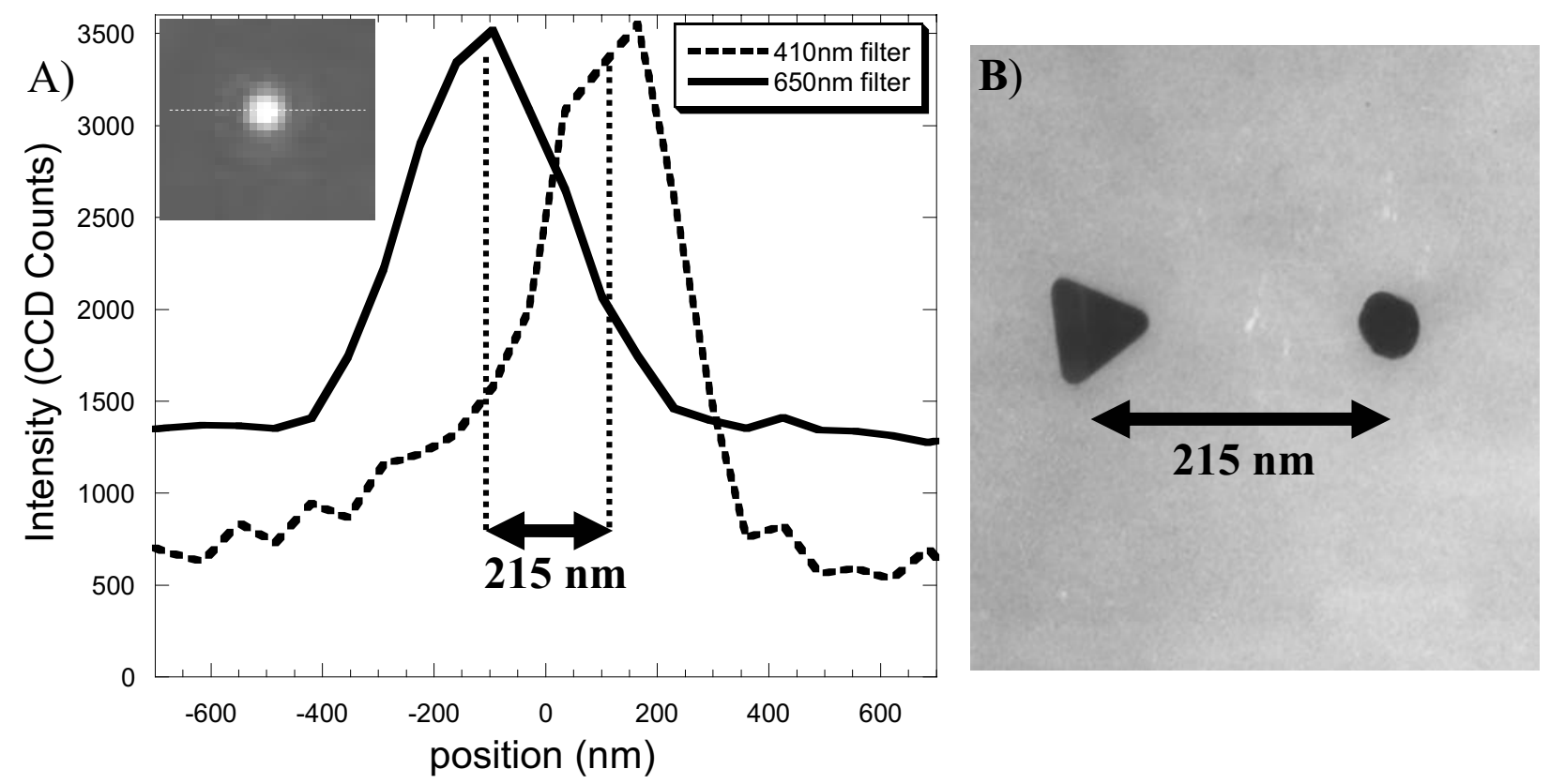

Figure 6: Filtered imaging of closely spaced plasmon resonant nanoparticles. A) Pixel intensity linescan through point source image taken of plasmon resonant object with both $410 \mathrm{~nm}$ and $650 \mathrm{~nm}(10 \mathrm{~nm}$ bandpass) filtered illumination, using the P.I. CCD and 50x objective. Inset: CCD Image of the pair under white light illumination. The dashed line is the direction of the linescan shown. B) TEM image of the same particle pair with the same orientation as the line-scan plot. 
useful to have other isolated PRPs in the image area to use as fiduciary markers should the substrate move between each filtered image, These PRP markers also act as a calibration of the microscope magnification and sample orientation relative to fiduciaries when determined by TEM. The peak position of the diffraction limited point source is dominated by the sphere at $410 \mathrm{~nm}$ and by the tetrahedron at $650 \mathrm{~nm}$, causing the two linescans to have individual peak positions separated by the physical spacing of the two nanoparticles in agreement with the TEM.

Bandpass filtering can also be used to enhance the imaging resolution of different sections on a composite plasmon resonant nanowire. The plasmon resonant nanowires we describe here are fabricated using a templated plating technique, and can be composed of arbitrary lengths of silver, gold and nickel $[6,13]$. Each $25 \mathrm{~nm}$ to $40 \mathrm{~nm}$ diameter metal nanorod has a unique polarization-dependent spectral signature. For example, under white light illumination polarized along the nanowire short axis, silver preferentially scatters blue light, gold scatters green, and nickel scatters white light. Therefore, a short segment of silver embedded in a gold nanowire is distinguishable by its unique blue response. Filtered imaging of the composite nanowire enables the location and characterization of segments smaller than the diffraction limit of light. Figure 6 illustrates the use of a 10nm bandpass filter centered at $410 \mathrm{~nm}$ to highlight the scattering of a short silver segment embedded in a gold nanowire. As described above, a pixel intensity line-scan is used to establish the relative position of the segment. This technique becomes more important as the density of coding on the plasmon resonant nanowires, and possibly other plasmonic devices, increases.
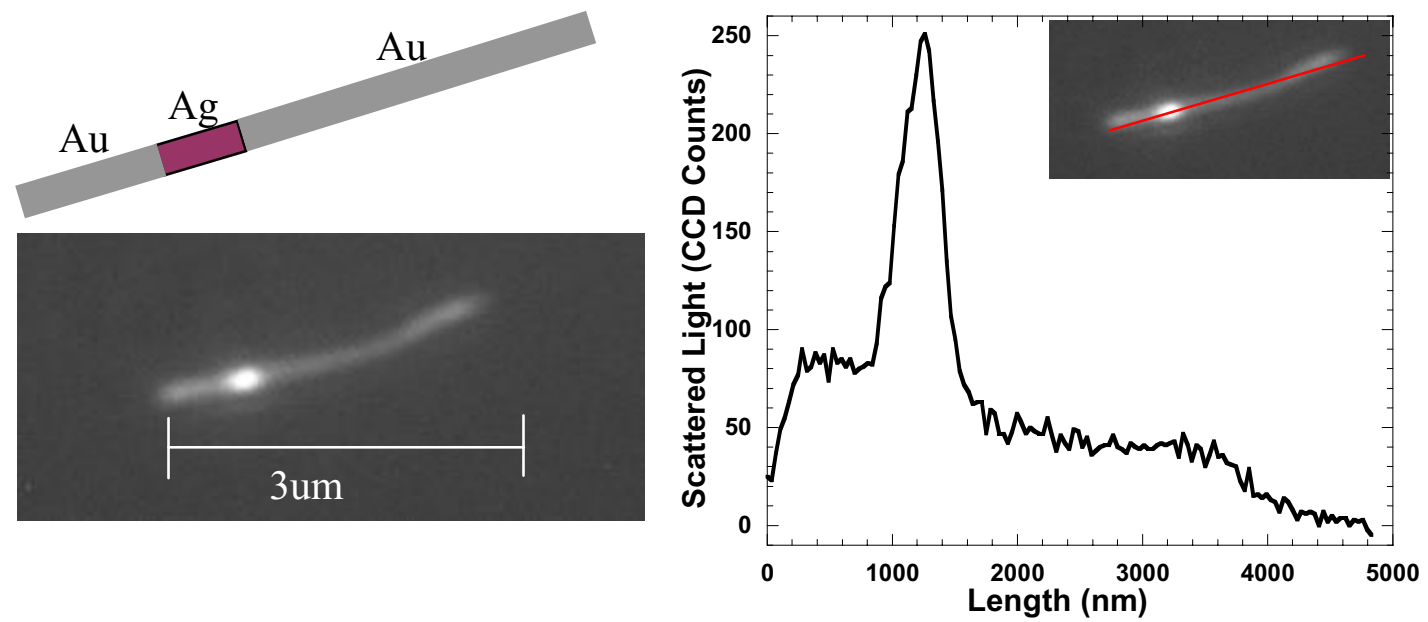

Figure 6: $10 \mathrm{~nm}$ bandpass (centered at $410 \mathrm{~nm}$ ) filtered illumination (with polarization perpendicular to rod length) of composite $(\sim 30 \mathrm{~nm}$ diameter gold/silver/gold) plasmon resonant nanowire. Linescan through CCD image displays greater scattering from the silver segment relative to the gold nanowire at this wavelength, allowing for improved spatial resolution of the silver segment.

\subsection{Substrate Effect on Plasmon Resonance}

The scattering properties of PRPs are particularly sensitive to nearby scattering objects, including the substrate on which they are deposited. The substrate properties generally shift the plasmon resonance wavelength, and alter the far-field scattering profile. For an extensive analysis of the effect of the substrate on the resonance wavelength of PRPs see reference [7]. Here, we will briefly discuss the far field scattering profile only. We have used a wide range of substrates, including glass slides, silicon wafers and formvar films on TEM grids. Those substrates that are highly conductive (or lossy) have a striking impact on the PRP point spread function. The scattering response of a PRP can be simply envisioned as being formed from the radiation of two dipoles excited in the PRP, one parallel to the substrate and one perpendicular to the substrate. The parallel dipole of a PRP placed on a conductive surface will be reduced by its image dipole, while the perpendicular dipole will be enhanced by the image dipole. We thus find that PRPs deposited on HF cleaned silicon wafers, metal films and other conductive substrates image as "donuts"; that is, the central maximum in the point spread function is missing. Figure 7a shows the scattering from an $80 \mathrm{~nm}$ gold colloid on silicon (with a $100 \mathrm{~nm}$ $\mathrm{Si}_{2}$ layer), along with the pixel intensity line-cut through an optical CCD image of the particle. Figure $7 \mathrm{~b}$ shows the same for an $80 \mathrm{~nm}$ gold colloid on conductive Hf cleaned silicon. The parallel dipole component, which is damped on a conductive surface, is related to the horizontal physical dimension of the plasmon resonant nanostructure. Thus, substrate effect must be accounted for in the interpretation of optical signatures from nanoparticles on surfaces. This is 
especially important for the case of asymmetric nanostructures, such as those created by lithographic techniques on conductive surfaces, where the strong dipole response may be significantly modified by the substrate.
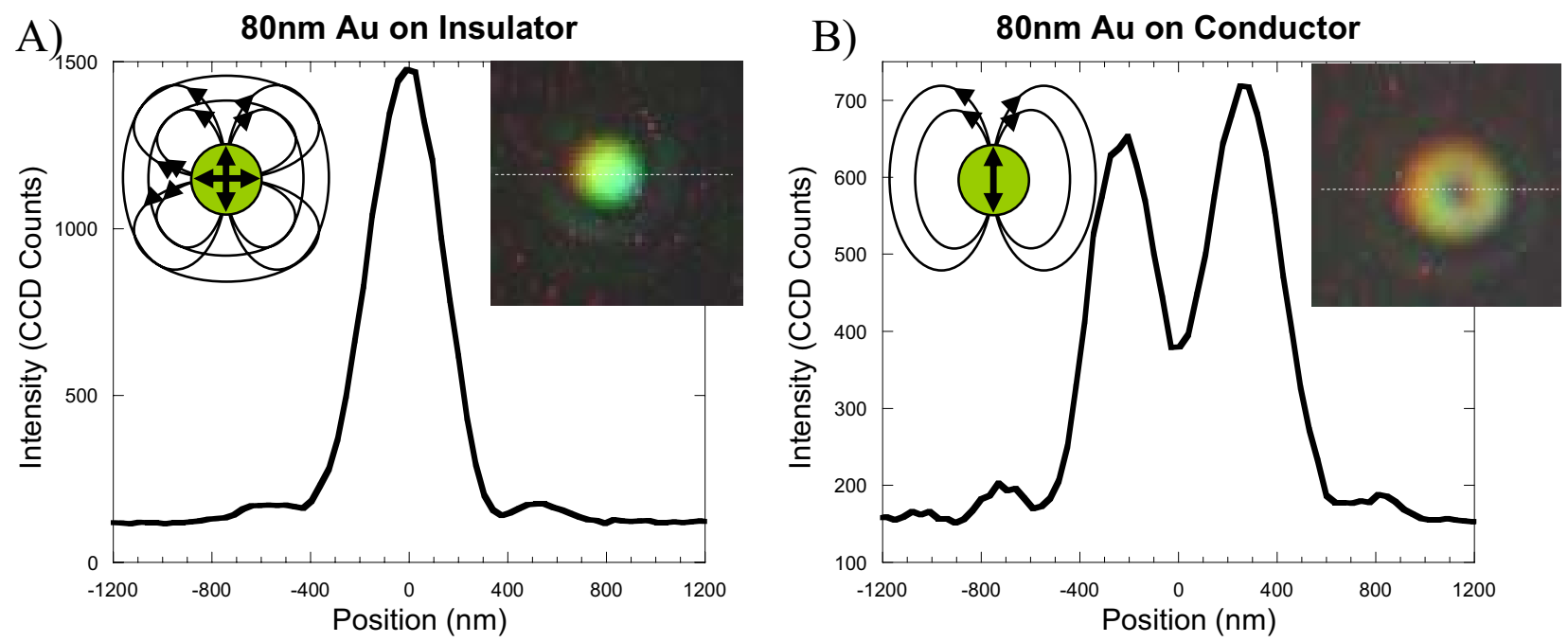

Figure 7: Color images (Coolpix 995) and P.I. CCD intensity linescans through the far field optical CCD images from: A) 80nm gold colloid on $100 \mathrm{~nm} \mathrm{SiO}_{2}$ on silicon wafer. B) $80 \mathrm{~nm}$ gold colloid on Hf cleaned silicon wafer. Inset drawings indicate the simple 2dipole approximation of the far-field pattern in which the horizontal dipole is damped on the conductive substrate resulting in a donut shaped far field image.

\subsection{Index of Refraction of Surrounding Medium}

There has been considerable interest recently in utilizing the sensitivity of the plasmon resonance spectral peak of gold and silver nanoparticles as an indicator of the local dielectric environment. It has been clearly shown that the resonance
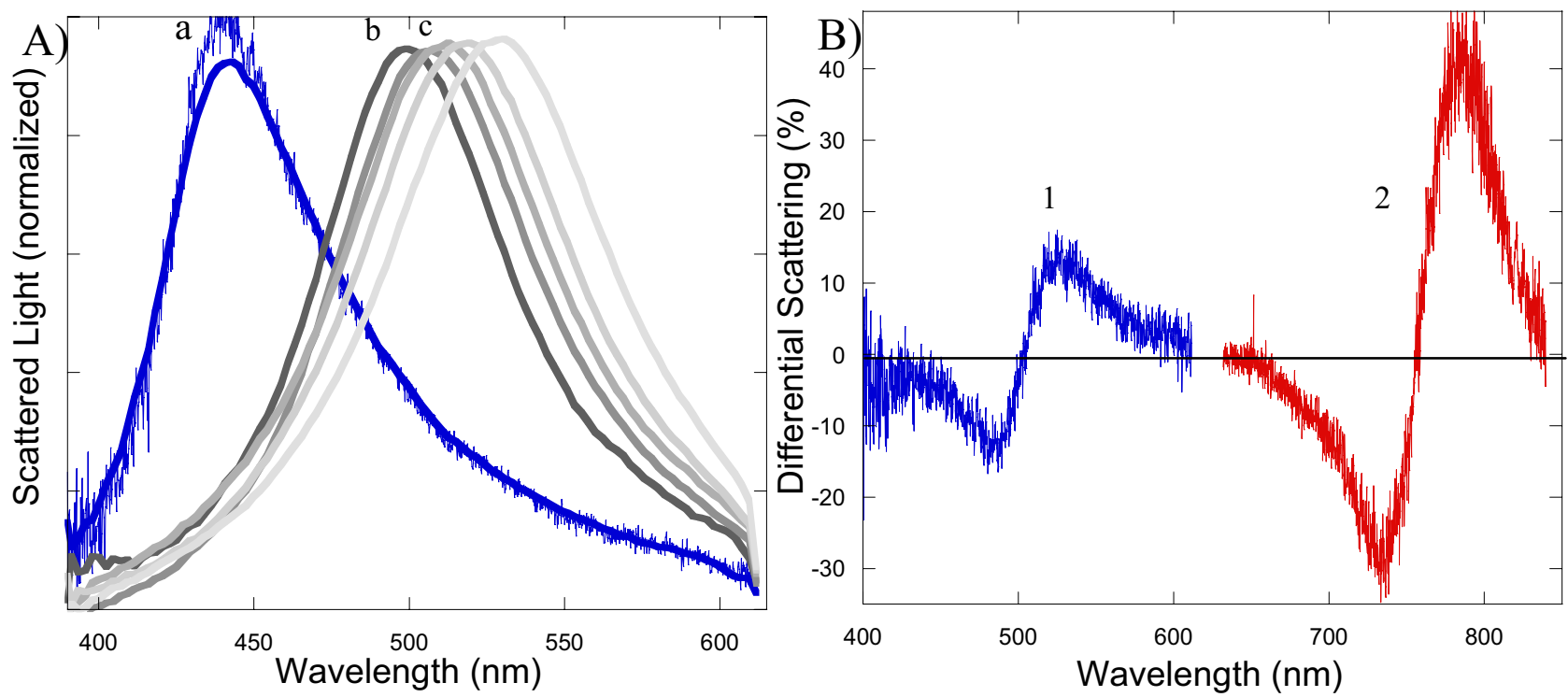

Figure 8: Plasmon Resonance Peak shift due to changing local dielectric index. A) Typical blue (spherical) silver PRP Resonance curve as it is shifted from a) air, to b) 1.44 index, to c) 1.48 index, to further incremental index increases of 0.04 . B) Differential scattering \% (spectrum in 1.48 index - spectrum in 1.44 index) of 1) blue (spherical) silver PRP, and 2) typical red (tetrahedron) silver plasmon resonant particle. 
shift from a very small local dielectric change caused by a biomolecule binding can be readily measured from both ensembles of PRPs [24-26] and more recently from individual PRPs [23]. We have made numerous spectral measurements of the effect that a change in local dielectric has on individual PRPs [14]. Instead of binding biomolecules to the surface of PRPs, we have incrementally altered the surrounding index using index oils. The index oils are applied, the spectral measurements on individual PRPs are made, and then oil is removed by a brief rinse in Xylene. We have found that the incremental shift caused by 0.04 in index of refraction can be readily observed from individual silver PRPs. Furthermore, we have found that the sensitivity to the changing index is dependent on the initial shape and color of the PRP. Figure 8A represents the typical plasmon resonance peak red-shift from a blue (spherical) particle as it is brought from a local index of 1.0 to a local index of 1.44, 1.48, 1.52, 1.56 and 1.6. Figure 8B represents the typical differential (i.e. spectrum in 1.48 index - spectrum in 1.44 index) resonance shift from the same blue (spherical) PRP and from a red (tetrahedron) as they undergo a local dielectric change from 1.44 to 1.48. Note: we have applied the differential spectrum analytical technique which is used by Sonnischien et al in reference [23] to highlight the differential shift for a 0.04 index oil change and to display the increased sensitivity characteristic of tetrahedron shaped PRPs. It is clear that single plasmon resonant nanoparticles can be used effectively as highly miniaturized biosensors, and that by studying the characteristics of the individual particles, we can potentially tune the response to maximize the sensitivity.

\section{CONCLUSION}

We have demonstrated that, using the proper optical microscope apparatus, plasmon resonant nanostructures can be studied individually and they are bright optical scatterers. The mapping of dark field optical images and spectra of silver and/or gold spheres, tetrahedrons, pentagons, and nanorods, to their TEM determined geometry has shown the dependence of the plasmon resonance to their shape and size. In addition, the plasmon resonance of these nanoparticles is highly sensitive to their local dielectric environment. This structure-environment relationship will provide the basis for a variety of future photonic applications.

\section{ACKNOWLEDGEMENTS}

We would like to thank The National Center for Microscopy and Imaging Research (NCMIR) at UCSD, and Andy Pommer of the UCSD Physics Machine Shop. We would also like to acknowledge the support of the NSF DBI 9876651, NIH PHS HG0195901, the NIH SBIR R43GM62097, and the Lounsbery Foundation.

\section{REFERENCES}

1. J.J. Mock, M. Barbic, D. Smith, D. Schultz, S. Schultz (2002). "Shape Effects in Plasmon Resonance of Individual Colloidal Silver Nanoparticles." Journal of Chemical Physics 116 6755-6759 (2002).

2. Rongchao Jin, YunWei Cao, Chad A. Mirkin, K.L. Kelly, George C. Schatz, and J.G. Zheng, "Photoinduced Conversion of Silver Nanospheres to Nanoprisms." Science 294: 1901-1903 (2001).

3. Sihai Chen and David L. Carroll, "Synthesis and Characterization of Truncated Triangular Silver Nanoplates." Nanoletters 2 1003-1007 (2002).

4. Isabel Pastoriza-Santos and Luis M. Liz-Marzan, "Synthesis of Silver Nanoprisms in DMF". Nanoletters 2 903905 (2002).

5. J. R. Krenn, G. Schider, W. Rechberger, B. Lamprecht, A. Leitner and F. R. Aussenegg "Design of multipolar plasmon excitations in silver nanoparticles.” Applied Physics Letters 77 3379-3381 (2000).

6. M. Barbic, J.J. Mock, and S. Schultz (2002). "Single Crystal Silver Nanowires Prepared by the Metal Amplification Method." Journal of Applied Physics $919341-9345$ (2002)

7. K. Lance Kelly, Eduardo Coronado, Lin Lin Zhao, and George C. Schatz, "The Optical Properties of Metal Nanoparticles." J. Phys. Chem. B. 107 668-677 (2003).

8. J. D. Kottman, O. J. F. Martin, D. R. Smith and S. Schultz, "Scattering properties of nanoparticles with arbitrary shape." Chem. Phys. Lett. 341 1-3 (2001). 
9. Sihai Chen, Zhiyong Fan, and David L. Carroll, "Silver Nanodisks: Synthesis, Characterization, and Self Assembly." J. of Phys. Chem. B. 106 10777-10781 (2002).

10. C. Sonnichsen, T Franzl, T Wilk, G von Plessen, an d J Feldman, " Plasmon resonances in large noble-metal clusters." New Journal of Physics 4 93.1-93.8 (2002).

11. Xiao-Hong Nancy Xu, Jun Chen, Robert B. Jeffers, and Sophia Kyriacou, "Direct measurement of sizes and dynamics of single living membrane transporters using nanooptics." Nanoletters 2 175-182 (2002).

12. Michael P. Mallin and Catherine J. Murphy, "Solution-Phase Synthesis of Sub-10 nm Au-Ag Alloy Nanoparticles." Nanoletters 2 1235-1237 (2002).

13. J.J. Mock, S.J. Oldenburg, D.R. Smith, D.A. Schultz, S. Schultz, "Composite Plasmon Resonant Nanowires." Nanoletters 2 465-469 (2002).

14. Jack J. Mock, David R. Smith, and Sheldon Schultz, "Local Refractive Index Dependence of Plasmon Resonance Spectra from Individual Nanoparticles." Nanoletters 3, 485-491 (2003).

15. Traci R. Jensen, Michelle Duval Malinsky, Lance Kelly, Anne A. Lazarides, George C. Schatz, and Richard P. Van Duyne, "Nanosphere Lithography: Effect of the External Dielectric Medium on the Surface Plasmon Resonance of a Periodic Array of Silver Nanoparticles." J. Phys. Chem. B 103, 9846-9853 (1999).

16. C. Sonnichsen, S. Geier, N. E. Hecker, G. von Plessen, J. Feldmann, H. Ditlbacher, B. Lamprecht, J. R. Krenn, F. R. Aussenegg, V. Z-H. Chan, J. P. Spatz, and M. Moller, "Spectroscopy of single metallic nanoparticles using total internal reflection microscopy." Applied Physics Letters 77, 2949-2951 (2000).

17. K.-H. Su, Q.-H. Wei, X. Zhang, J.J. Mock, D.R. Smith, and S. Schultz, "Interparticle Coupling Effects on Plasmon Resonances of Nanogold Partticles.” Nanoletters (In Press).

18. W. Rechberger, A. Hohenau, A. Leitner, J.R. Krenn, B Lamprecht, and F.R. Aussenegg, "Optical properties of two interacting gold nanoparticles." Optics Communications 220 137-141 (2003).

19. Hiroharu Tamaru, Hitoshi Kuwata, Hideki T. Miyazaki, Kenjiro Miyano, "Resonant light scattering from individual Ag nanoparticles and particle pairs." Applied Physics Letters 80 1826-1828 (2002).

20. Nordin Felidj, Jean Aubard, and Georges Levi, "Discrete dipole approximation for ultraviolet-visible extinction spectra simulation of silver and gold colloids." J. of Chem. Phys. 111 1195-1208 (1999).

21. H. Ditlbacher, J. R. Krenn, B. Lamprecht, A. Leitner, and F. R. Aussenegg, "Spectrally coded optical data storage by metal nanoparticles." Optics Letters 25 563-565 (2000).

22. S. Schultz, D.R. Smith, J.J. Mock, and D.A. Schultz, "Single molecule detection with nonbleaching multicolor optical immunolabels." Proc. Natl. Acad. Sci. U.S.A. 97 996-1001 (2000).

23. G. Raschke, S. Kowarik, T. Franzl, C. Sonnichsen, T.A. Klar, and J. Feldman, "Biomolecular Recognition Based on Single Gold Nanoparticle Light Scattering." Nanoletters (2003).

24. Amanda J. Haes and Richard P. Van Duyne," A Nanoscale Optical Biosensor: Sensitivity and Selectivity of an Approach Based on the Localized Surface Plasmon Resonance Spectroscopy of Triangular Silver Nanoparticles." JACS 124, 10596-10604 (2002).

25. Nidhi Nath and Ashutosh Chilkoti, "A Colorimetric Gold Nanoparticle Sensor to Interrogate Biomolecular Interactions in Real Time on a Surface." Anal. Chem. 74 504-509 (2002).

26. Takayuki Okamoto, Ichirou Yamaguchi and Tetsushi Kobayashi, "Local plasmon sensor with gold colloid monolayers deposited upon glass substrates." Optics Letters 25 372-374 (2000).

27. M. Quinten, A. Leitner, J. R. Krenn, and F. R. Aussenegg, "Electromagnetic energy transport via linear chains of silver nanoparticles." Optics Letters 23 1331-1333 (1998).

28. H. Ditlbacher, J. R. Krenn, G. Schider, A. Leitner, and F. R. Aussenegg, "Two-dimensional optics with surface plasmon polaritons." Applied Physics Letters 81 1762-1764 (2002).

29. C. Chicanne, T. David, R. Quidant, J. C. Weeber, Y. Lacroute, E. Bourillot, A. Dereux, G. Colas des Francs and C. Girard, "Imaging the Local Density of States of Optic Corals." Physical Review Letters 88 097402-1 (2002).

30. Stefan A. Maier, Pieter G. Kik, and Harry A. Atwater, "Observation of coupled plasmon-polariton modes in Au nanoparticle chain waveguides of different lengths: Estimation of waveguide loss." Applied Physics Letters $\mathbf{8 1}$ 1714-1716 (2002).

31. Yoshihisa Ohko, Tetsu Tatsuma, Tsoyushi Fujii, Kenji Naoi, Chisa Niwa, Yoshinobu Kubota, and Akira Fujishima, "Multicolor photochroism of $\mathrm{TiO}_{2}$ films loaded with silver nanoparticles." Nature Materials 2, 2931 (2003).

32. D.A. Schultz, "Plasmon resonant particles for biological detection." Current Opinion in Biotechnology 14, 13$22(2003)$. 